\title{
INTERSECTION THEORY OF LINEAR EMBEDDINGS
}

\author{
SEAN KEEL
}

ABSTRACT. We study intersection theoretic properties of subschemes defined by ideal sheaves of linear type in particular their behavior with respect to blowups and segre classes.

\section{INTRODUCTION}

Micali [M] has shown that if an ideal $I$ in a ring $A$ is generated by a regular sequence, then the canonical surjection from the $d$ th symmetric power of $I$ to $I^{d}$

$$
\operatorname{Sym}_{A}^{d}(I) \rightarrow I^{d}
$$

is an isomorphism for all $d$. Huneke [H] calls an ideal for which (1) holds an "ideal of linear type." Valla [V] gives numerous criteria for when an ideal is of linear type, and has shown in particular that this class of ideals contains ideals generated by a $d$-sequence as well as ideals of almost complete intersections. Such ideals can be quite general, for example Huneke shows in $[\mathrm{H}]$ that any ideal in a normal ring which is generated by two elements can be generated by a $d$-sequence. Further references, supplied by the referee, are included in the bibliography.

In this paper we investigate subschemes defined by ideal sheaves of linear type. A subscheme $X \stackrel{i}{\hookrightarrow} Y$ with ideal sheaf $I \subset \mathscr{O}_{Y}$ is said to be linearly embedded if the ideal sheaf is of linear type. If (1) is an isomorphism only for $d$ sufficiently large, then the embedding is said to be weakly linear.

We will show that a number of properties of regular embeddings extend to the more general class of linear embeddings. Throughout the discussion we will be concerned with a composition of embeddings:

$$
X \stackrel{i}{\hookrightarrow} Y \stackrel{j}{\hookrightarrow} Z
$$

with $J \subset I \subset \mathscr{O}_{Z}$ the ideal sheaves of $Y$ and $X$ respectively.

If $X \stackrel{i}{\hookrightarrow} Y$ and $Y \stackrel{j}{\hookrightarrow} Z$ are regular embeddings, then there is an exact sequence of normal bundles:

$$
0 \rightarrow \mathbf{N}_{X} Y \rightarrow \mathbf{N}_{X} Z \rightarrow i^{*} \mathbf{N}_{Y} Z \rightarrow 0
$$

which implies the equation among Chern classes

$$
c\left(\mathbf{N}_{X} Z\right)=c\left(\mathbf{N}_{X} Y\right) \cdot c\left(i^{*} \mathbf{N}_{Y} Z\right) .
$$

Received by the editors August 1, 1989 and, in revised form February 22, 1990.

1980 Mathematics Subject Classification (1985 Revision). Primary 14C17. 
This equation can be rewritten as

$$
s(X, Y)=c\left(i^{*} \mathbf{N}_{Y} Z\right) \cap s(X, Z)
$$

or as

$$
i^{*} s(Y, Z)=c\left(\mathbf{N}_{X} Y\right) \cap s(X, Z)
$$

where for any subscheme $X \subset Y, s(X, Y)$ is the segre class of $X$ in $Y$. For a regular embedding

$$
s(X, Y)=c\left(\mathbf{N}_{X} Y\right)^{-1} \cap[X] .
$$

(2) makes sense even if $i$ is not a regular embedding so long as $j$ is regular (i.e. both sides of the equality are defined) while (3) makes sense even if $j$ is not a regular embedding so long as $i$ is regular. However, it is known that under these conditions the equalities do not always hold (see [F, p. 139]). We show that (2) holds provided $i$ is a linear embedding and $j$ is regular and that (3) holds provided $j \circ i$ is a linear embeddings and $i$ is regular.

Let $\widetilde{Z} \stackrel{\pi}{\rightarrow} Z$ be the blowup of $Z$ along $X$ and let $\tilde{Y}$ be the blowup of $Y$ along $X . \tilde{Y}$ embeds in $\tilde{Z}$ as the strict transform of $Y$ under $\tilde{Z} \stackrel{\pi}{\rightarrow} Z$. Let $E=\pi^{-1}(X)$ be the exceptional divisor of the blowup. $E$ is a subscheme of $\pi^{-1}(Y)$. Let $\mathscr{R}=\mathscr{R}\left(E, \pi^{-1}(Y)\right)$ be the residual scheme. (This notation is fixed throughout the section.) (In general, if we have a scheme $Y$ with a subscheme $X$ and a cartier divisor $D$ of $Y$ which is itself a subscheme of $X$

$$
D \hookrightarrow X \hookrightarrow Y
$$

then we obtain a scheme $\mathscr{R}=\mathscr{R}(D, X)$ the residual scheme to $D$ in $X$, by locally dividing equations for $X$ by a defining function for $D$. In terms of the ideal sheaves, $\mathscr{R}$ is characterized by the equation

$$
\mathscr{I}_{\mathscr{R}} \cdot \mathscr{I}_{D}=\mathscr{I}_{X} \text {.) }
$$

If $i$ and $j$ are regular embeddings it is known (Fulton [F, p. 169]) that

(1) $\widetilde{Y}=\mathscr{R}$,

(2) $\widetilde{Y} \hookrightarrow \widetilde{Z}$ is a regular embedding,

(3) $\mathbf{N}_{\widetilde{Y}} \widetilde{Z}=\pi^{*}\left(\mathbf{N}_{Y} Z\right) \otimes \mathscr{O}(-E)$.

We show that these results hold as long as $X \stackrel{i}{\hookrightarrow} Y$ is a linear embedding and $Y \stackrel{j}{\hookrightarrow} Z$ is a regular embedding.

In summary our main results are the following three theorems: (notation as above)

Theorem 1. Consider the following conditions:

(1) $X \stackrel{i}{\hookrightarrow} Y$ is a weakly linear embedding,

(2) $\mathscr{R}=\tilde{Y}$,

(3) $I^{n} \cap J=I^{n-1} \cdot J$ for all $n$ sufficiently large.

Then $(1) \Rightarrow(2) \Leftrightarrow(3)$ and if $X \stackrel{j \cdot i}{\hookrightarrow} Z$ is weakly linear, then $(1) \Leftrightarrow(2)$. Furthermore, if

$$
X \stackrel{i}{\hookrightarrow} Y
$$

is linear then

$$
I^{n} \cap J=I^{n-1} \cdot J
$$


for all $n$ and if

$$
X \stackrel{j \circ i}{\leftrightarrow} Z
$$

is linear then the two statements are equivalent.

Theorem 2. If $i$ is a linear embedding and $j$ is a regular embedding then

(1) $\widetilde{Y}=\mathscr{R}$,

(2) $\widetilde{Y} \hookrightarrow \widetilde{Z}$ is a regular embedding,

(3) $\mathbf{N}_{\widetilde{Y}} \tilde{Z}=\pi^{*}\left(\mathbf{N}_{Y} Z\right) \otimes \mathscr{O}(-E)$,

(4) There exists a cone

$$
C \rightarrow X \times \mathbf{A}^{1}
$$

flat over $\mathbf{A}^{1}$ with fibers $C_{t}$ equal to $C_{X} Z$ for $t$ not equal to zero and $C_{0}$ equal to $C_{X} Y \oplus i^{*} N_{Y} Z$.

(5) $s(X, Y)=c\left(i^{*} \mathbf{N}_{Y} Z\right) \cap s(X, Z)$.

Theorem 3. If $i$ is regular and $j \circ i$ is linear, then

(1) There exists a cone

$$
C \rightarrow X \times \mathbf{A}^{1}
$$

flat over $\mathbf{A}^{1}$ with fibers $C_{t}$ equal to $C_{X} Z$ for $t$ not equal to zero and $C_{0}$ equal to $N_{X} Y \oplus i^{*} C_{Y} Z$.

(2) The embedding

$$
i^{*} C_{Y} Z \hookrightarrow C_{Y} Z
$$

is regular of the same codimension as $i$.

(3)

$$
i^{*} s(Y, Z)=c\left(\mathbf{N}_{X} Y\right) \cap s(X, Z) .
$$

These results are applied in [K1 and $\mathrm{K} 2]$ where they are used to study the intersection theory of parameterizing spaces for polygons in projective space and the intersection theory of the moduli space of stable pointed curves of genus zero.

I wish to thank W. Fulton, S. Bloch, M. Murthy, M. Kumar, and G. Lubeznik for a number of interesting discussions. I also wish to thank J. Sally for bringing to my attention the works of Huneke and Valla and for encouragement in general.

This paper is organized as follows: Section 1 recalls some standard notations and facts. Section 2 is a catalogue of results from Valla [V] which will be used. Section 3 contains the proofs of Theorems 1, 2 and 3. Section 4 gives examples and counterexamples, and the appendix contains new "geometric" proofs of several of the results of Valla, as well as a lemma clarifying the relationship between linear and weakly linear.

\section{NOTATIONS AND CONVENTIONS}

Here we collect basic notation and facts which will be used throughout the chapter. We continue to follow the notation of the introduction. All spaces considered are noetherian equidimensional schemes. $\operatorname{Pow}_{A}(I)$ denotes the Rees algebra of $I$

$$
\operatorname{Pow}_{A}(I)=A \oplus I \oplus I^{2} \oplus I^{3} \cdots .
$$

$\operatorname{gr}_{A}(I)$ denotes the associated graded algebra

$$
\operatorname{gr}_{A}(I)=A / I \oplus I / I^{2} \oplus I^{2} / I^{3} \cdots
$$


$C_{X} Y$ denotes the normal cone to $X$ in $Y$, i.e.

$$
C_{X} Y=\operatorname{Specgr}_{\mathscr{O}_{Y}}(I) \text {. }
$$

When $X$ is regularly embedded this cone is a bundle, and in that case we denote it by $N_{X} Y$.

For a commutative ring $A$ and an $A$ module $M, \operatorname{Sym}_{A}(M)$ is the symmetric algebra of $M$,

$$
\operatorname{Sym}_{A}(M)=A \oplus \operatorname{Sym}_{A}^{1}(M) \oplus \operatorname{Sym}_{A}^{2}(M) \oplus \cdots .
$$

Some properties of Sym: If $N$ is a submodule of $M$, we have an exact sequence

$$
N \otimes \operatorname{Sym}_{A}^{d-1}(M) \rightarrow \operatorname{Sym}_{A}^{d}(M) \rightarrow \operatorname{Sym}_{A}^{d}\left(\frac{M}{N}\right) \rightarrow 0 .
$$

In particular,

$$
\operatorname{Sym}_{A}(M) \otimes_{A} \frac{A}{I}=\operatorname{Sym}_{A / I}\left(\frac{M}{I \cdot M}\right) .
$$

Also

$$
\operatorname{Sym}_{A}^{d}(N \oplus M)=\bigoplus_{i+j=d} \operatorname{Sym}_{A}^{i}(N) \otimes_{A} \operatorname{Sym}_{A}^{j}(M) .
$$

We will make frequent use of the following: If $S$ is a sheaf of graded algebras then there is a canonical identification of subschemes of $\operatorname{Proj}(S)$ and graded ideals of $S$ via

$$
I \subset S \leadsto \operatorname{Proj}\left(\frac{S}{I}\right) .
$$

Under this identification, two ideals $I$ and $J$ give the same subscheme iff their graded components $I_{d}$ and $J_{d}$ are equal for all $d$ sufficiently large. In addition there is a correspondence between quasicoherent $\mathscr{O}_{\operatorname{Proj}(S)}$ modules and sheaves of graded $S$ modules

$$
M \rightsquigarrow \widetilde{M} .
$$

(See $[\mathrm{M}]$.

\section{Catalogue of results of Valla}

Here we record some results of Valla [V] which will be useful. We will refer to the results by their numbering in $[\mathrm{V}]$ preceded by a $\mathrm{V}$.

Theorem V1.3. $\operatorname{Sym}_{\mathscr{O}_{Y}}(I)$ is isomorphic to $\operatorname{Pow}_{A}(I)$ iff $\operatorname{Sym}_{\mathscr{O}_{X}}\left(I / I^{2}\right)$ is isomorphic to $\operatorname{gr}_{A}(I)$.

In the appendix we give a geometric proof of this (see Theorem A.2) as well as of the related result:

$\operatorname{Proj}\left(\operatorname{Sym}_{\mathscr{Q}_{Y}}(I)\right) \cong \operatorname{Proj}\left(\bigoplus_{d}\left(I^{d}\right)\right) \Leftrightarrow \operatorname{Proj}\left(\operatorname{Sym}_{\mathscr{Q}_{X}}\left(\frac{I}{I^{2}}\right)\right) \cong \operatorname{proj}\left(\bigoplus_{d}\left(\frac{I^{d}}{I^{d+1}}\right)\right)$

(see Theorem A.1).

Theorem V2.1. If $i$ is linear and $j$ is regular then $j \circ i$ is linear.

Remark. A geometric proof of this fact is given in the appendix (see Theorem A.3). 
Theorem V2.3. If $i$ is a regular embedding and $i \circ j$ is a linear embedding then $j$ is linear along $X$ (i.e. the ideal $J_{x} \subset \mathscr{O}_{Z, x}$ is of linear type for each $x$ in $X)$.

Remark. A geometric proof of this fact is given in the appendix (see Theorem A.4).

Proofs of Theorems 1-3. We now prove Theorems 1-3 in succession. Before proceeding to Theorem 1, we obtain the following lemma:

Lemma 1. $\mathscr{R}$ is the subscheme of $\tilde{Z}$ defined by the graded ideal sheaf

$$
\bigoplus_{n} I^{n-1} \cdot J \subset \bigoplus_{n} I^{n}
$$

Proof. As remarked in the introduction $\mathscr{R}$ is characterized by the fact that

$$
\mathscr{I}_{\mathscr{R}} \cdot \mathscr{I}_{E}=\mathscr{I}_{\pi^{-1}(Y)}
$$

The ideal sheaf of $E=\pi^{-1}(X)$ is

$$
\bigoplus_{n} I^{n+1}=I \cdot \mathscr{O}_{\widetilde{Z}}
$$

while the graded ideal sheaf of $\pi^{-1}(Y)$ is $\bigoplus_{n} J \cdot I^{n}$. From our remark the result is now clear.

Theorem 1. Consider the following conditions:

(1) $X \stackrel{i}{\leftrightarrow} Y$ is a weakly linear embedding,

(2) $\mathscr{R}=\tilde{Y}$,

(3) $I^{d} \cap J=I^{n-1} \cdot J$ for all $n$ sufficiently large.

Then $(1) \Rightarrow(2) \Leftrightarrow(3)$ and if $X \stackrel{j \cdot i}{\hookrightarrow} Z$ is weakly linear, then $(1) \Leftrightarrow(2)$. Furthermore: If $X \stackrel{i}{\leftrightarrow} Y$ is linear then $I^{n} \cap J=I^{n-1} \cdot J$ for all $n$ and if $X \stackrel{\text { joi }}{\hookrightarrow} Z$ is linear then the two statements are equivalent.

Proof. We have a commutative diagram

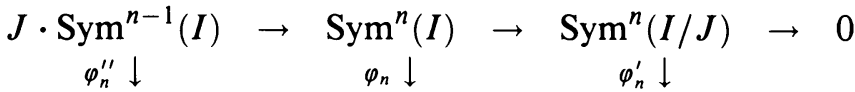

$$
\begin{aligned}
& 0 \rightarrow J \cap I^{n} \quad \rightarrow \quad I^{n} \rightarrow(I / J)^{n}
\end{aligned}
$$

with $\varphi_{n}$ surjective and $J \cdot I^{n-1}$ the image of $\varphi_{n}^{\prime \prime}$. (1) is equivalent to $\varphi_{n}^{\prime}$ being injective for $n$ sufficiently large. The graded ideal sheaf of $\tilde{Y}$ is

$$
\bigoplus_{n} J \cap I^{n} \subset \bigoplus_{n} I^{n}
$$

so by the preceding lemma, (2) is equivalent to $\varphi_{n}^{\prime \prime}$ being surjective for $n$ large which in turn is equivalent to (3). Now by the snake lemma, (1) implies (2). If $j \circ i$ is weakly linear, then $\varphi_{n}$ is an isomorphism for $n$ sufficiently large, and so again by the snake lemma, (1) and (2) are equivalent. The proof of the second pair of assertions is analogous.

Remark. The proof also shows that when $j \circ i$ is weakly linear

$$
\mathscr{R}=\operatorname{Proj}\left(\operatorname{Sym}_{\mathscr{O}_{Y}}(I / J)\right) \text {. }
$$

Kleinman has made this observation in the case $j \circ i$ is regular (see [KL]).

The proof of Theorem 2 will require the following: 
Lemma. Let $X, D$ be subschemes of a scheme $Y$ with $D$ a cartier divisor, $D \cap X \hookrightarrow X$ a cartier divisor and $D \cap X \hookrightarrow X$ a linear embedding. Then if

$$
\mathbf{B} L(Y, X) \stackrel{\pi}{\rightarrow} Y
$$

is the blowup of $Y$ along $X$ then $\pi^{-1}(D)$ is the blowup of $D$ along $D \cap X$.

Proof. Since $\mathbf{B} L(D, D \cap X)$ embeds canonically in $\pi^{-1}(D)$ it is enough to check the theorem locally on $Y$ along $X \cap D$. Thus we may assume that $Y$ is $\operatorname{Spec}(A)$ for $(A, m)$ a local noetherian ring, that $X$ is defined by an ideal $I \subset m$ and that $f \in m$ defines $D$. Then by Lemma A.4 $X$ is linearly embedded. Thus

$$
\begin{aligned}
\mathbf{B} L(Y, X) & =\operatorname{Proj}\left(\operatorname{Sym}_{A}(I)\right), \\
\mathbf{B} L(D, D \cap X) & =\operatorname{Proj}\left(\operatorname{Sym}_{A / f}(I / I \cap(f))\right), \\
\pi^{-1}(D) & =\operatorname{Proj}\left(\operatorname{Sym}_{A / f}(I / I \cdot f)\right) .
\end{aligned}
$$

$I \cdot f$ is equal to $I \cap(f)$ since $f$ is not a zero divisor modulo $I$. This completes the proof.

Theorem 2. If $i$ is a linear embedding and $j$ is a regular embedding then

(1) $\tilde{Y}=\mathscr{R}$,

(2) $\widetilde{Y} \hookrightarrow \widetilde{Z}$ is a regular embedding,

(3) $\mathbf{N}_{\widetilde{Y}} \widetilde{Z}=\pi^{*}\left(\mathbf{N}_{Y} Z\right) \otimes \mathscr{O}(-E)$,

(4) there exists a cone

$$
C \rightarrow X \times \mathbf{A}^{1}
$$

flat over $\mathbf{A}^{1}$ with fibres $C_{t}$ equal to $C_{X} Z$ for $t$ not equal to zero and $C_{0}$ equal to $C_{X} Y \oplus i^{*} N_{Y} Z$.

(5) $s(X, Y)=c\left(i^{*} \mathbf{N}_{Y} Z\right) \cap s(X, Z)$.

Proof. By Theorem V1.3 $j \circ i$ is linear and so (1) holds by Theorem 1. By [F, p. 164] there is a canonical embedding of cones

$$
C_{\mathscr{R}} \tilde{Z} \hookrightarrow \pi^{*} N_{Y} Z \otimes \mathscr{O}(-E) \text {. }
$$

Thus if we establish (2), it will follow that $C_{\mathscr{R}} \tilde{Z}$ is a vector bundle and (since both bundles will have the same rank) that $(*)$ is an isomorphism, establishing (3). Also (4) implies (5) by [F, p. 234]. We establish (4) by means of the deformation to the normal cone. By [F, p. 64] there is a scheme $S$ (in the language of [F] $S$ is $M^{0}$ ) with a flat map $S \stackrel{\pi}{\rightarrow} \mathbf{A}^{1}$ and an embedding

$$
X \times \mathbf{A}^{1} \stackrel{K}{\hookrightarrow} S
$$

commuting with the maps to $\mathbf{A}^{1}$ with the following properties:

The open set

$$
U=\pi^{-1}\left(\mathbf{A}^{1} \backslash\{0\}\right)
$$

is isomorphic to the product $Z \times\left(\mathbf{A}^{1} \backslash\{0\}\right)$ and the embedding $X \times\left(\mathbf{A}^{1} \backslash\{0\}\right) \hookrightarrow$ $U$ is the composition

$$
X \times\left(\mathbf{A}^{1} \backslash\{0\}\right) \hookrightarrow Y \times\left(\mathbf{A}^{1} \backslash\{0\}\right) \hookrightarrow Z \times\left(\mathbf{A}^{1} \backslash\{0\}\right) .
$$

The fiber over $0, S_{0}$ is isomorphic to the normal bundle of $Y$ in $Z$ and the embedding $K_{0}$ is the composition

$$
X \stackrel{i}{\hookrightarrow} Y \hookrightarrow N_{Y} Z
$$


where the last embedding is the zero section. ( $S$ is an open subset of the blowup of $Z \times \mathbf{A}^{1}$ along $Y \times\{0\}$.)

We will show that $C=C_{X \times \mathrm{A}^{1}} S$ has the properties prescribed by (4).

Let $L$ be $\mathbf{A}^{1}$ and let $B$ be the blowup of $S \times L$ along $X \times \mathbf{A}^{1} \times\{0\}$, let $E$ be the exceptional divisor of this blowup and let $p$ and $q$ be the compositions

$$
p: B \rightarrow S \times \mathbf{A}^{1} \stackrel{p_{1}}{\rightarrow} S \stackrel{\pi}{\rightarrow} \mathbf{A}^{1}, \quad q: E \hookrightarrow B \rightarrow \mathbf{A}^{1} .
$$

Of course $E$ is $\mathbf{P}(C \oplus 1)$.

Observe that $p^{-1}\left(\mathbf{A}^{1} \backslash 0\right)$ is the product

$$
\begin{gathered}
\mathbf{B} L\left(Z \times \mathbf{A}^{1} \backslash\{0\} \times L, X \times \mathbf{A}^{1} \backslash\{0\} \times\{0\}\right) \\
=\mathbf{B} L(Z \times L, X \times\{0\}) \times \mathbf{A}^{1} \backslash 0
\end{gathered}
$$

and that $q^{-1}\left(\mathbf{A}^{1} \backslash 0\right)$ is the product

$$
\mathbf{P}\left(C_{X} Z \oplus 1\right) \times \mathbf{A}^{1} \backslash 0 .
$$

By the preceding lemma (applied to the subschemes $X \times\{0\}$ and $S_{0} \times L$ of $S \times L) p^{-1}(0)$ is the blowup of $S_{0} \times \mathbf{A}^{1}$ along $X$ and $q^{-1}(0)$ is the exceptional divisor of this blowup. Thus if $f$ is a local parameter for $\mathbf{A}^{1}$ at zero and $g$ is a local equation for $E$ in $\mathscr{O}_{B}$ near some point of the fibre $p^{-1}(0)$ then $(f, g)$ is a regular sequence. Hence $(g, f)$ is a regular sequence and $q$ is flat, near the point we are considering. Letting the point vary over the fibre we conclude that $q$ is flat. From what we have seen it follows that

$$
\begin{aligned}
q^{-1}(t) & =\mathbf{P}\left(C_{X} Z \oplus 1\right) \text { for } t \neq 0, \\
q^{-1}(0) & =\mathbf{P}\left(C_{X \times 0}\left(N_{Y} Z \times \mathbf{A}^{1}\right)\right)=\mathbf{P}\left(C_{X} Y \oplus i^{*} N_{Y} Z \oplus 1\right) .
\end{aligned}
$$

In particular $C$ is flat over $\mathbf{A}^{1}$ and has the desired fibres.

In order to establish (2) it is sufficient to demonstrate that the embedding

$$
\mathbf{P}\left(C_{X} Y\right)=\tilde{Y} \cap E \hookrightarrow E=\mathbf{P}\left(C_{X} Z\right)
$$

is regular. (Since $j$ is regular, the embedding $\tilde{Y} \hookrightarrow \tilde{Z}$ is regular away from $E$. $E \cap \tilde{Y}$ is a cartier divisor on $\tilde{Y}$ and $E$ is a cartier divisor on $\widetilde{Z}$. The reduction then follows from the fact that a regular sequence remains regular when the elements are permuted.)

The question is local and we may assume that $Z$ is $\operatorname{sr}: 2 \mathrm{c}(A)$ and $J=$ $\left(j_{1}, j_{2}, \ldots, j_{d}\right)$ with $j_{1}, j_{2}, \ldots, j_{d}$ a regular sequence in $A$.

We induct on $d$. Using V1.3 we may assume that $d=1$ and $J=(f)$. In order to show that the embedding is regular it suffices to show that the image of $f$ in $I / I^{2}$ (as an element of degree one) is a nonzero divisor in $\operatorname{gr}_{A}(I)$. Since $f$ is a nonzero divisor it suffices to show that

$$
f \cdot I^{k} \cap I^{k+2} \subset f \cdot I^{k+1} \text { for all } k .
$$

This holds by Theorem 1. This completes the proof of the theorem.

Our proof of Theorem 3 will require the following lemma:

Lemma 2. Let $(A, m)$ be a local noetherian ring. Let $J \subset m$ be an ideal and $x \in m$ an element whose image in $A / J$ is a nonzero divisor. If the ideal $J+(x)$ is of linear type then $x$ is a nonzero divisor and

$$
x \cdot J^{d-1} \cap J^{d}=x \cdot J^{d} \quad \text { for all } d .
$$


Proof. By Theorem V2.3 $J$ is of linear type. Since the image of $x$ is not a zero divisor in $A / J$ we have an exact sequence

$$
0 \rightarrow J \stackrel{(- \text { id }, \cdot x)}{\longrightarrow} A \oplus J \stackrel{\cdot x+\text { id }}{\longrightarrow}(x+J) \rightarrow 0 .
$$

This induces the exact sequence of symmetric groups

$$
\bigoplus_{i=0}^{d-1} \operatorname{Sym}^{i}(J) \otimes J \stackrel{f}{\rightarrow} \oplus \bigoplus_{i=0}^{d} \operatorname{Sym}^{i}(J) \stackrel{g}{\rightarrow} \operatorname{Sym}^{d}(x+J) \rightarrow 0 .
$$

Here $g$ is induced by

$$
\operatorname{Sym}^{i}(J) \stackrel{\bar{\otimes} x}{\rightarrow} \bar{\otimes}^{-i} \operatorname{Sym}^{d}(x+J)
$$

(where $\bar{\otimes}$ indicates multiplication in the symmetric algebra) and $f$ is induced by

$$
\operatorname{Sym}^{i}(J) \otimes J \stackrel{\left(f_{1}, f_{2}\right)}{\rightarrow} \operatorname{Sym}^{i}(J) \oplus \operatorname{Sym}^{i+1}(J)
$$

where $f_{1}$ is induced by $A$ module multiplication $\alpha \otimes j \rightarrow-\alpha \cdot j$ and $f_{2}$ is induced by $\alpha \otimes j \rightarrow \alpha \bar{\otimes}(j \cdot x)$. We have a commutative diagram

$$
\begin{array}{ccccccc}
\bigoplus_{i=0}^{d-1} \operatorname{Sym}_{h}^{i}(J) \otimes J & \stackrel{f}{\rightarrow} & \bigoplus_{i=0}^{d} \operatorname{Sym}^{i}(J) & \stackrel{g}{\rightarrow} & \operatorname{Sym}^{d}(x+J) & \rightarrow & 0 \\
q \downarrow & & & & p \downarrow & & \\
\bigoplus_{i=0}^{d-1} J^{i+1} & \stackrel{f^{\prime}}{\rightarrow} & \bigoplus_{i=0}^{d} J^{i} & \stackrel{g^{\prime}}{\rightarrow} & (x+J)^{d} & \rightarrow & 0 .
\end{array}
$$

$h$ is surjective and $q$ and $p$ are isomorphisms (since $J$ and $(J+x)$ are of linear type). Here $g^{\prime}$ is induced by

$$
J^{i} \stackrel{x^{d-i}}{\rightarrow}(x+J)^{d}
$$

and $f^{\prime}$ is induced by

$$
J^{i+1} \stackrel{(-\mathrm{id}, \cdot x)}{\rightarrow} J^{i} \oplus J^{i+1} .
$$

In particular we have the exact sequence

$$
0 \rightarrow J^{d} \stackrel{(- \text { id }, \cdot x)}{\rightarrow} J^{d-1} \oplus J^{d} \stackrel{\cdot x+\text { id }}{\rightarrow}\left(x \cdot J^{d-1}+J^{d}\right) \rightarrow 0 .
$$

This sequence implies that

$$
x \cdot J^{d-1} \cap J^{d}=x \cdot J^{d}
$$

and

$$
\operatorname{Ann}(x) \cap J^{d-1}=\operatorname{Ann}(x) \cap J^{d}
$$

for all $d$. This second equality implies that

$$
\operatorname{Ann}(x) \subset J^{d} \text { for all } d
$$

which implies that $\operatorname{Ann}(x)$ is zero.

Remark. The conclusion of the lemma is equivalent to the statement that $x$ is a nonzero divisor modulo $J^{d}$ for all $d$.

Lemma 2 has the following corollary which is included as it gives a sort of generalization of the fact that in a local ring a permutation of a regular sequence is a regular sequence. The corollary is not needed for the proof of Theorem 3. 
Corollary. Let $(A, m)$ be a local noetherian ring. Let $J \subset m$ be an ideal and $x_{1}, x_{2}, \ldots, x_{n}$ a sequence of elements whose image in $A / J$ is a regular sequence. If the ideal

$$
J+\left(x_{1}, x_{2}, \ldots, x_{n}\right)
$$

is of linear type then $J$ is of linear type and $x_{1}, \ldots, x_{n}$ is a regular sequence (in $A$ ).

Proof. $J$ is of linear type by Theorem V2.3. We will prove the second assertion by induction on $n$. The case of $n=1$ is contained in Lemma 2. In general the remark following Lemma 2 implies that $x_{n}$ is a nonzero divisor modulo $\left(J+\left(x_{1}, \ldots, x_{n-1}\right)\right)^{d}$ for all $d$. Also by Theorem V2.3 $J+\left(x_{1}, \ldots, x_{n-1}\right)$ is an ideal of linear type. It then follows by Lemma A.5 that $J+\left(x_{1}, \ldots, x_{n-1}\right)+$ $\left(x_{n}\right) /\left(x_{n}\right)$ is an ideal of linear type in $A / x_{n}$. But since $x_{1}, \ldots, x_{n-1}$ is a regular sequence modulo $J+\left(x_{n}\right) /\left(x_{n}\right)$ it follows by induction that $x_{1}, \ldots, x_{n-1}$ is a regular sequence modulo $x_{n}$. This completes the proof.

Theorem 3. If $i$ is regular and $j \circ i$ is linear, then

(1) There exists a cone $C \rightarrow X \times \mathbf{A}^{1}$ flat over $\mathbf{A}^{1}$ with fibres $C_{t}$ equal to $C_{X} Z$ for $t$ not equal to zero and $C_{0}$ equal to $N_{X} Y \oplus i^{*} C_{Y} Z$.

(2) The embedding

$$
i^{*} C_{Y} Z \hookrightarrow C_{Y} Z
$$

is regular of the same codimension as $i$.

$$
i^{*} s(Y, Z)=c\left(\mathbf{N}_{X} Y\right) \cap s(X, Z) .
$$

Proof. We have an exact sequence of $\mathscr{O}_{X}$ modules

$$
0 \rightarrow \frac{J}{J \cap I^{2}} \rightarrow \frac{I}{I^{2}} \rightarrow \frac{I}{I^{2}+J} \rightarrow 0
$$

with $I / I^{2}+J$ locally free. By [F, p. 73, Example 4.1.7] this implies an equality of segre classes

$$
s\left(\frac{J}{J \cap I^{2}}\right)=c\left(\frac{I}{I^{2}+J}\right) \cap s\left(\frac{I}{I^{2}}\right) .
$$

(For an $\mathscr{O}_{X}$ module $\mathscr{F}, s(\mathscr{F})$ is the segre class of the cone $\operatorname{Spec}(\operatorname{Sym}(\mathscr{F}))$. )

$$
\operatorname{Spec}\left(\operatorname{Sym}_{\mathscr{O}_{X}}\left(I / I^{2}+J\right)\right)
$$

is the normal bundle of $X$ in $Y$ and

$$
\operatorname{Spec}\left(\operatorname{Sym}_{\mathscr{O}_{X}}\left(I / I^{2}\right)\right)
$$

is the normal cone of $X$ in $Z$. Also, by Theorem V2.3 the embedding $j$ is linear along $X$ (i.e. $J_{x} \subset \mathscr{O}_{Z, x}$ is an ideal of linear type for each $x$ in $X$ ) and thus

$$
\begin{aligned}
\operatorname{gr}_{\mathscr{O}_{z}}(J) \otimes \mathscr{O}_{X} & =\operatorname{Sym}_{\mathscr{O}_{Y}}\left(J / J^{2}\right) \otimes \mathscr{O}_{X} \\
& =\operatorname{Sym}_{\mathscr{O}_{Y}}(J / J \cdot I) \\
& =\operatorname{Sym}_{\mathscr{O}_{Y}}\left(J / J \cap I^{2}\right)
\end{aligned}
$$

(this last equality holds by Theorem 1). With these observations our segre class equality becomes

$$
s\left(i^{*} C_{Y} Z\right)=c\left(N_{X} Y\right) \cap s\left(C_{X} Z\right)=c\left(N_{X} Y\right) \cap s(X, Z) .
$$


In addition our exact sequence of $\mathscr{O}_{X}$ modules implies the exact sequence of cones (in the sense of [F, p. 73])

$$
0 \rightarrow N_{X} Y \rightarrow C_{X} Z \rightarrow i^{*} C_{Y} Z \rightarrow 0 .
$$

By [F, p. 73] this implies (1). Thus to establish (3) it suffices to demonstrate that

$$
s\left(i^{*} C_{Y} Z\right)=i^{*} s\left(C_{Y} Z\right) .
$$

We have a fiber diagram

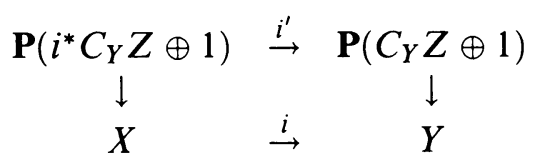

and the result will follow if we can show that $i_{*}^{\prime}$ is a regular embedding of the same codimension as $i$. Of course this will also yield (2). This follows from the next lemma by replacing $X Y$ and $Z$ by $X \times\{0\} Y \times\{0\}$ and $Z \times \mathbf{A}^{1}$. The new triple continue to satisfy the assumptions of the lemma by Theorem V1.3 (or Lemma A.3).

Lemma 3. If $i$ is a regular embedding and $j \circ i$ is a linear embedding then

$$
\mathbf{P}\left(i^{*} C_{Y} Z\right) \hookrightarrow \mathbf{P}\left(C_{Y} Z\right)
$$

is a regular embedding of the same codimension as $i$.

Proof. We have a fiber diagram

$$
\begin{array}{ccc}
\mathbf{P}\left(i^{*} C_{Y} Z\right) & \rightarrow & \mathbf{P}\left(C_{Y} Z\right) \\
\downarrow & & \downarrow \\
X & \rightarrow & Y .
\end{array}
$$

The question is local on $X$ and so we may assume that $Z$ is the spectrum of a local ring. Let $n$ be the codimension of $i$. We prove the lemma by induction on $n$.

Case $n=1$. We can assume that $I=J+(x)$ with $x$ a nonzero divisor modulo $J$. Then by the previous lemma we conclude that $x$ is a nonzero divisor and

$$
x \cdot J^{d-1} \cap J^{d}=x \cdot J^{d} .
$$

Together these imply that $x$ (as an element of degree zero) is a nonzero divisor in $\operatorname{gr}_{A}(J)$ which in turn implies the result.

General Case. Factor $i$ as a composition of two regular embeddings

$$
X \stackrel{i_{1}}{\hookrightarrow} X_{1} \stackrel{i_{2}}{\hookrightarrow} Y
$$

each of codimension less than $n$. By induction the embedding $i_{2}^{*} C_{Y} Z \hookrightarrow C_{Y} Z$ is regular of the same codimension as $i_{2}$ (observe that $j \circ i_{2}$ is linear by V2.3). Thus it suffices to show that the embedding $i^{*} C_{Y} Z \hookrightarrow i_{2}^{*} C_{Y} Z$ is regular of the same codimension as $i_{1}$. We have a fiber diagram

$$
\begin{array}{ccc}
i_{1}^{*} C_{X_{1}} Z & \hookrightarrow & C_{X_{1}} Z \\
\downarrow & & \downarrow \\
i^{*} C_{Y} Z & \hookrightarrow & i_{2}^{*} C_{Y} Z .
\end{array}
$$


By induction the top row is a regular embedding of the same codimension as $i_{1}$. The next lemma implies that the right column is a smooth map and thus the result follows.

Lemma. If $i$ is regular and $j \circ i$ is linear then the canonical map $C_{X} Z \rightarrow i^{*} C_{Y} Z$ is smooth.

Proof (as in the proof of Theorem 3). We have an exact sequence of $\mathscr{O}_{X}$ modules

$$
0 \rightarrow \frac{J}{J \cap I^{2}} \rightarrow \frac{I}{I^{2}} \rightarrow \frac{I}{I^{2}+J} \rightarrow 0
$$

with $I / I^{2}+J$ locally free, $\operatorname{Sym}_{\mathscr{O}_{X}}\left(I / I^{2}\right)$ equal to $C_{X} Z$ and $\operatorname{Sym}_{\mathscr{O}_{X}}\left(J / J \cap I^{2}\right)$ equal to $i^{*} C_{Y} Z$. The result is local on $X$ and so we can assume that $I / I^{2}+J$ is isomorphic to $\mathscr{O}_{X}^{n}$ for some $n$ and the above exact sequence is split. The result then follows from the fact that for an $A$ module $M$ the symmetric algebra $\operatorname{Sym}_{A}\left(M \oplus A^{n}\right)$ is a polynomial ring over $\operatorname{Sym}_{A}(M)$.

Counterexamples. The purpose of this section is to prove that the following two "Theorems" are false for a sequence of embeddings $X \stackrel{i}{\hookrightarrow} Y \stackrel{j}{\hookrightarrow} Z$ with $i$ regular and $j$ linear.

(1) $j \circ i$ is weakly linear.

(2) $i^{*} s(Y, Z)=c\left(N_{X} Y\right) \cap s(X, Z)$.

In order to construct counterexamples we need the following lemmas:

Lemma 1. The embedding of a point in a scheme is weakly linear if and only if the scheme is regular at the point.

Proof. Let $(A, m)$ be the local ring at the point. If the embedding is weakly linear then the graded map

$$
\operatorname{Sym}_{A / m}\left(m / m^{2}\right) \rightarrow A / m \oplus m / m^{2} \oplus \cdots
$$

is an isomorphism for high degree. This implies (for example by considering the Hilbert polynomial) that $A$ is regular.

Lemma 2. Let $x, y, z, w$ be the coordinates of $\mathbf{A}^{4}$. The embedding of the plane $x=z=0$ in the cone $x y=z w$ is linear.

Proof. Let $A$ be the coordinate ring of the cone

$$
A=\frac{k[x, y, z, w]}{x y=z w}
$$

and let $I$ be the ideal defining the plane $I=(x, z)$. Since $A$ is normal and $I$ is generated by two elements this follows from the result of Huneke stated in the introduction. Just for fun we give a direct proof. Let $B$ be the quotient of the polynomial ring

$$
\frac{A[X, Z]}{(x Z-z X, X y-Z w)} \text {. }
$$

There is a surjection $B \rightarrow \operatorname{Sym}_{A}(I)$ defined by sending $X$ and $Z$ to the elements $x$ and $z$ in $\operatorname{Sym}_{A}^{1}(I)$. We will show that $B / I \rightarrow \operatorname{gr}_{A}(I)$ is an isomorphism. This will imply in particular that the surjection

$$
\operatorname{Sym}_{A / I}\left(I / I^{2}\right) \rightarrow \operatorname{gr}_{A}(I)
$$


is an isomorphism and thus imply the result by Theorem V1.3. Since $B / I$ is an integral domain it is enough to show that the surjection

$$
(B / I)_{y} \rightarrow \operatorname{gr}_{A_{y}}\left(I_{y}\right)
$$

is an isomorphism. $B_{y}$ is isomorphic to the polynomial ring $A_{y}[Z]$ and in particular is a domain of dimension three. $\operatorname{Pow}_{A_{y}}\left(I_{y}\right)$ is a domain of the same dimension and hence the surjection $B_{y} \rightarrow \operatorname{Pow}_{A_{y}}\left(I_{y}\right)$ is an isomorphism. This implies the desired result.

For our counterexamples we take $Z, Y$ and $X$ to be the affine cone $x y=$ $z w$, the plane $x=z=0$ and the vertex of the cone $x=y=z=w=0$.

$i$ is regular and $j$ is linear by Lemma 2 but $j \circ i$ is not weakly linear by Lemma 1. This contradicts (1).

$$
c\left(N_{X} Y\right) \cap s(X, Z)=s(X, Z)=2 \cdot[X] .
$$

By Theorem 3.2

$$
s(Y, Z)=c\left(N_{Z} \mathbf{A}^{4}\right)^{-1} \cap s\left(Y, \mathbf{A}^{4}\right)=s\left(Y, \mathbf{A}^{4}\right)=[Y] .
$$

Thus $i^{*}(s(Y, Z))=[X]$. This contradicts $(2)$.

APPENDIX: "GeOMETRIC" PROOFS

Theorem A.1. The embedding

$$
\operatorname{Proj}\left(\bigoplus_{n} I^{n}\right) \hookrightarrow \operatorname{Proj}\left(\operatorname{Sym}_{\mathscr{O}_{Y}}(I)\right)
$$

is an isomorphism if and only if the embedding

$$
\operatorname{Proj}\left(\bigoplus_{n} I^{n} / I^{n+1}\right) \hookrightarrow \operatorname{Proj}\left(\operatorname{Sym}_{\mathscr{O}_{X}}\left(I / I^{2}\right)\right)
$$

is an isomorphism.

Remark. Of course Theorem A.1 is equivalent to the statement:

Algebraic formulation. The following are equivalent:

(1) $\operatorname{Sym}_{A}^{d}(I)$ is isomorphic to $I^{d}$ for all $d$ sufficiently large.

(2) $\operatorname{Sym}_{A / I}^{d}\left(I / I^{2}\right)$ is isomorphic to $I^{d} / I^{d+1}$ for all $d$ sufficiently large.

The proof will require two lemmas:

Lemma A.1. The subscheme

$$
\operatorname{Proj}\left(\operatorname{Sym}_{\mathscr{O}_{X}}\left(I / I^{2}\right)\right) \hookrightarrow \operatorname{Proj}\left(\operatorname{Sym}_{\mathscr{O}_{Y}}(I)\right)
$$

is locally principal (i.e. the ideal sheaf is locally generated by a single element). $\operatorname{Proof}$ (G. Lubeznik). We may assume that $Y$ is $\operatorname{Spec}(A)$ and that $X$ is defined by the ideal $I=\left(i_{1}, i_{2}, \ldots, i_{m}\right)$. Let $\overline{i_{j}}$ be the element $i_{j} \in \operatorname{Sym}_{A}^{1}(I)$.

Then $\operatorname{Proj}\left(\operatorname{Sym}_{\mathscr{O}_{Y}}(I)\right)$ is covered by the affine open sets

$$
U_{j}=\operatorname{Spec}\left(\operatorname{Sym}_{A}(I)_{\left(\overline{i_{j}}\right)}\right)
$$

where $\operatorname{Sym}_{A}(I)_{\left(\overline{i_{j}}\right)}$ is the subring of $\operatorname{Sym}_{A}(I)_{\bar{i}_{j}}$ consisting of elements of degree 
zero. In $U_{j} \operatorname{Proj}\left(\operatorname{Sym}_{\mathscr{O}_{X}}\left(I / I^{2}\right)\right)$ is defined by the ideal $I \subset A=\operatorname{Sym}_{A}^{0}(I)$. In the ring $\operatorname{Sym}_{A}(I)_{\overline{i_{j}}} i_{k}=\left(\overline{i_{k}} / \overline{i_{j}}\right) \cdot i_{j}$. Thus $i_{j}$ generates $I$ in $U_{j}$. This completes the proof.

Lemma A.2. Let $D \hookrightarrow Y \hookrightarrow Y^{\prime}$ be a composition of subschemes of $Y^{\prime}$ such that $D \hookrightarrow Y$ is a cartier divisor of $Y$ and $D \hookrightarrow Y^{\prime}$ is a locally principal subscheme of $Y^{\prime}$. If $Y \backslash D$ is equal to $Y^{\prime} \backslash D$ then $Y$ is equal to $Y^{\prime}$.

In order to prove the lemma it is enough to establish the following local result:

Local Version. Let $(A, m)$ be a local noetherian ring. Let $f \in m$ be an element of $A$. If $I \subset(f)$ is an ideal such that the image of $f$ is a nonzero divisor in $A / I$ then $I$ is the zero ideal.

Proof. We have an exact sequence

$$
0 \rightarrow I \rightarrow A \rightarrow A / I \rightarrow 0 .
$$

The sequence remains exact after tensoring with $A /(f)$ since by assumption $f$ is not a zero divisor in $A / I$. Thus we have an exact sequence

$$
0 \rightarrow I / f \cdot I \rightarrow A / f \rightarrow A /(I, f) \rightarrow 0 .
$$

Since $I$ is contained in $f A / f \rightarrow A /(I, f)$ is an isomorphism and so $I$ is equal to $f \cdot I$. The result then follows from Nakayama's lemma.

Proof of Theorem A.1. Assume that the embedding

$$
\operatorname{Proj}\left(\bigoplus_{n} I^{n} / I^{n+1}\right) \hookrightarrow \operatorname{Proj}\left(\operatorname{Sym}_{\mathscr{O}_{X}}\left(I / I^{2}\right)\right)
$$

is an isomorphism. Then by Lemma A.1 we have a composition of subschemes

$$
\operatorname{Proj}\left(\bigoplus_{n} I^{n} / I^{n+1}\right) \hookrightarrow \operatorname{Proj}\left(\bigoplus_{n} I^{n}\right) \hookrightarrow \operatorname{Proj}\left(\operatorname{Sym}_{\mathscr{O}_{Y}}(I)\right)
$$

such that

$$
\operatorname{Proj}\left(\bigoplus_{n} I^{n} / I^{n+1}\right) \hookrightarrow \operatorname{Proj}\left(\bigoplus_{n} I^{n}\right)
$$

is a cartier divisor and

$$
\operatorname{Proj}\left(\bigoplus_{n} I^{n} / I^{n+1}\right) \hookrightarrow \operatorname{Proj}\left(\operatorname{Sym}_{\mathscr{O}_{Y}}(I)\right)
$$

is a locally principal subscheme. Since on the complement of $\operatorname{Proj}\left(\bigoplus_{n} I^{n} / I^{n+1}\right)$ both $\operatorname{Proj}\left(\bigoplus_{n} I^{n}\right)$ and $\operatorname{Proj}\left(\operatorname{Sym}_{\mathscr{C}_{Y}}(I)\right)$ are isomorphic to $Y \backslash X$ the result follows from Lemma A.2.

Theorem A.2. The following are equivalent:

(1) $\operatorname{Sym}_{A}(I)$ is isomorphic to $\operatorname{Pow}_{A}(I)$.

(2) $\operatorname{Sym}_{A / I}\left(I / I^{2}\right)$ is isomorphic to $\operatorname{gr}_{A}(I)$.

The proof of Theorem A. 2 requires two lemmas: 
Lemma A.3. The following are equivalent:

(1) $\operatorname{Sym}_{A}^{d}\left(I / I^{2}\right) \stackrel{\cong}{\rightrightarrows} I^{d} / I^{d+1}$ for all $d$.

(2) $\operatorname{Sym}_{A[T]}^{d}\left((I, T) /(I, T)^{2}\right) \stackrel{\cong}{\rightrightarrows}(I, T)^{d} /(I, T)^{d+1}$ for all $d$.

(3) $\operatorname{Sym}_{A[T]}^{d}\left((I, T) /(I, T)^{2}\right) \stackrel{\cong}{\rightarrow}(I, T)^{d} /(I, T)^{d+1}$ for all $d$ sufficiently large. Proof. $(I, T)^{k} /(I, T)^{k+1}$ is an $A / I$ module (i.e. it is annihilated by $T$ and by $I$ ) and there is a canonical isomorphism

$$
(I, T)^{k} /(I, T)^{k+1} \cong I^{k} / I^{k+1} \oplus I^{k} / I^{k-1} \cdot T \oplus \cdots \oplus A / I \cdot T^{k} .
$$

Consequently there is a canonical identification

$$
\operatorname{Sym}_{A[T]}^{d}\left((I, T) /(I, T)^{2}\right)=\operatorname{Sym}_{A}^{d}\left(I / I^{2} \oplus A \cdot T\right)=\bigoplus_{i=0}^{d} \operatorname{Sym}_{A}^{i}\left(I / I^{2}\right) \cdot T^{d-i}
$$

and the map

$$
\operatorname{Sym}_{A[T]}^{d}\left((I, T) /(I, T)^{2}\right) \rightarrow(I, T)^{d} /(I, T)^{d+1}
$$

is the direct sum of the maps

$$
\operatorname{Sym}_{A}^{i}\left(I / I^{2}\right) \cdot T^{d-i} \rightarrow I^{i} / I^{i+1} \cdot T^{d-i}
$$

for $i$ between zero and $d$. In particular we see that the map

$$
\operatorname{Sym}_{A[T]}^{k}\left((I, T) /(I, T)^{2}\right) \rightarrow(I, T)^{k} /(I, T)^{k+1}
$$

is an isomorphism if and only if the maps

$$
\operatorname{Sym}_{A}^{i}\left(I / I^{2}\right) \rightarrow I^{i} / I^{i+1} \text { for } i=0,1, \ldots, k
$$

are all isomorphism. This implies the result.

Lemma A.4. The canonical map of $A$ modules

$$
\bigoplus_{i+j=d} \operatorname{Sym}_{A}^{i}(I) \cdot T^{j} \rightarrow \operatorname{Sym}_{A[T]}^{d}((I, T)) \otimes_{A[T]} A[T] /\left(T^{d+1}\right)
$$

is an isomorphism.

Proof. We compute $\operatorname{Sym}_{A[T]}^{d}((I, T))$ from the exact sequence:

$$
0 \rightarrow(I \cdot T) \rightarrow I \cdot A[T] \oplus T \cdot A[T] \rightarrow(I, T) \rightarrow 0 .
$$

This induces the exact sequence

$$
\begin{aligned}
& \operatorname{Sym}_{A[T]}^{d-1}(I \cdot A[T] \oplus T \cdot A[T]) \otimes(I \cdot T) \\
& \quad \rightarrow \operatorname{Sym}_{A[T]}^{d}(I \cdot A[T] \oplus T \cdot A[T]) \rightarrow \operatorname{Sym}_{A[T]}^{d}((I, T)) \rightarrow 0 .
\end{aligned}
$$

When we tensor with $A[T] /\left(T^{d+1}\right)$ the middle term becomes (after some easy manipulations)

$$
\begin{aligned}
& \operatorname{Sym}_{A}^{d}(I) \oplus T \cdot\left(\operatorname{Sym}_{A}^{d}(I) \oplus \operatorname{Sym}_{A}^{d-1}(I)\right) \\
& \oplus \cdots \oplus T^{d} \cdot\left(\operatorname{Sym}_{A}^{d}(I) \oplus \cdots \oplus \operatorname{Sym}_{A}^{1}(I) \oplus A\right)
\end{aligned}
$$

and the left term becomes

$$
T \cdot\left(\operatorname{Sym}_{A}^{d-1}(I) \otimes_{A} I\right) \oplus \cdots \oplus T^{d} \cdot\left(\operatorname{Sym}_{A}^{d-1}(I) \oplus \cdots \oplus \operatorname{Sym}_{A}^{1}(I) \oplus A\right) \otimes_{A} I .
$$


The map between them is induced by the maps

$$
\operatorname{Sym}_{A}^{j}(I) \otimes_{A} I \stackrel{(f, g)}{\rightarrow} \operatorname{Sym}_{A}^{j}(I) \oplus \operatorname{Sym}_{A}^{j+1}(I)
$$

for $j=0,1, \ldots, d-1$. Here $f$ is the multiplication map

and $g$ is the surjection

$$
\operatorname{Sym}_{A}^{j}(I) \otimes_{A} I \stackrel{f}{\rightarrow} \operatorname{Sym}_{A}^{j}(I)
$$

$$
\operatorname{Sym}_{A}^{j}(I) \otimes_{A} I \stackrel{g}{\rightarrow} \operatorname{Sym}_{A}^{j+1}(I) .
$$

The lemma will be established if we demonstrate that the induced map

$$
\operatorname{Sym}_{A}^{j}(I) \rightarrow \frac{\operatorname{Sym}_{A}^{j}(I) \oplus \operatorname{Sym}_{A}^{j+1}(I)}{\operatorname{image}(f, g)}
$$

is an isomorphism. The map is surjective since $g$ is surjective. To show that the map is injective it is enough to show that the kernel of $g$ is contained in the kernel of $f$.

Claim. The kernel of $g$ is generated by elements of the form

$$
\overline{i_{1} \otimes \cdots \otimes i_{j}} \otimes i_{j+1}-\overline{i_{1} \otimes \cdots \otimes i_{j+1}} \otimes i_{j} .
$$

Here $\overline{i_{1} \otimes \cdots \otimes i_{j}}$ indicates the image of $i_{1} \otimes \cdots \otimes i_{j}$ in $\operatorname{Sym}_{A}^{j}(I)$.

Since these elements are obviously in the kernel of $f$ the result follows once we establish the claim.

Proof of Claim. We have a commutative diagram

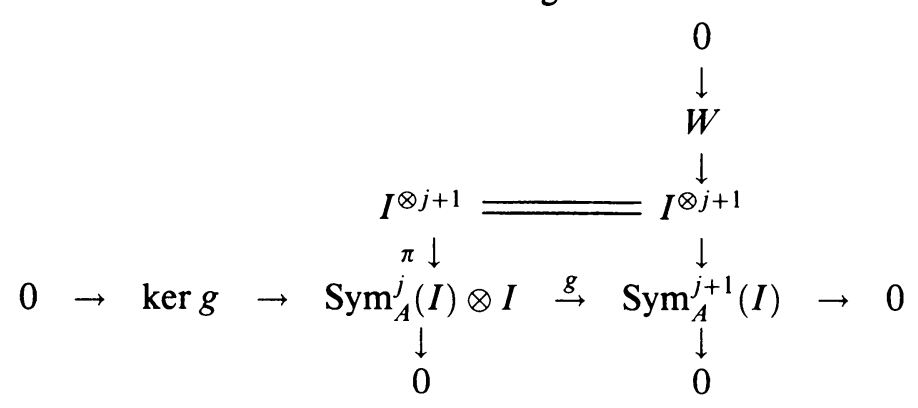

Here $\pi$ is the map obtained by tensoring the canonical surjection

$$
I^{\otimes j} \rightarrow \operatorname{Sym}_{A}^{j}(I)
$$

with $I$. By the Snake lemma the kernel of $g$ is the image under $\pi$ of $W$. W is generated by elements of the form

$$
i_{1} \otimes \cdots \otimes i_{k} \otimes \cdots \otimes i_{l} \otimes \cdots \otimes i_{j+1}-i_{1} \otimes \cdots \otimes i_{l} \otimes \cdots \otimes i_{k} \otimes \cdots \otimes i_{j+1} .
$$

These elements are in the kernel of $\pi$ except when one of $k$ or $l$ is $r+1$ and this collection of elements are exactly the generators proposed in the claim.

This completes the proof of the lemma.

Corollary. If $\operatorname{Sym}_{A[T]}^{d}((I, T))$ is isomorphic to $(I, T)^{d}$ then $\operatorname{Sym}_{A}^{j}(I)$ is isomorphic to $I^{j}$ for any $j$ less than or equal to $d$.

Proof of Corollary. We have a commutative diagram

$$
\begin{array}{ccc}
\operatorname{Sym}_{A}^{j}(I) & \stackrel{\cdot T^{d-j}}{\rightarrow} & \operatorname{Sym}_{A[T]}^{d}((I, T)) \\
\downarrow & & \downarrow \\
I^{j} & \cdot T^{d-j} & (I, T)^{d}
\end{array}
$$


By the previous lemma the top row is injective and thus if the right column is an isomorphism, the left column is injective and hence an isomorphism (in any case the left column is a surjection).

Proof of Theorem A.2. Assume that $\operatorname{Sym}_{A / I}\left(I / I^{2}\right)$ is isomorphic to $\operatorname{gr}_{A}(I)$. Then by Lemma A.3 we have that $\operatorname{Sym}_{A[T] /(I, T)}\left((I, T) /(I, T)^{2}\right)$ is isomorphic to $\operatorname{gr}_{A[T]}((I, T))$. Then by Theorem A.1 it follows that for all $d$ sufficiently large $\operatorname{Sym}_{A[T]}^{d}((I, T))$ is isomorphic to $(I, T)^{d}$. Finally, by the preceding corollary this implies that $\operatorname{Sym}_{A}^{j}(I)$ is isomorphic to $I^{j}$ for all $j$.

Corollary A.2. The following are equivalent:

(1) $X \hookrightarrow Y$ is a linear embedding.

(2) The composition

$$
X \times\{0\} \hookrightarrow Y \times\{0\} \hookrightarrow Y \times \mathbf{A}^{1}
$$

is a linear embedding.

(3) The composition

$$
X \times\{0\} \hookrightarrow Y \times\{0\} \hookrightarrow Y \times \mathbf{A}^{1}
$$

is a weakly linear embedding.

Proof. This is immediate from Theorem A.2 and Lemma A.3.

Lemma A.5. Let $(A, m)$ be a noetherian local ring. Let $I \subset m$ be an ideal of $A$ and let $f \in m$ be a nonzero divisor of $A$ whose image in $A / I$ is again a nonzero divisor. The following two conditions are equivalent:

(1) The surjection

$$
\operatorname{Sym}_{A /(f)}^{d}(I /(f) \cap I) \rightarrow I^{d} /(f) \cap I^{d}
$$

is an isomorphism.

(2) The surjection $\operatorname{Sym}_{A}^{d}(I) \rightarrow I^{d}$ is an isomorphism and the image of $f$ in $A / I^{d}$ is a nonzero divisor.

Proof. Observe that since the image of $f$ is not a zero divisor in $A / I$ the ideals $(f) \cap I$ and $f \cdot I$ are equal. We have a commutative diagram

$$
\begin{array}{ccc}
\operatorname{Sym}_{A}^{d}(I) \otimes_{A} A /(f) & \stackrel{l}{\rightarrow} & I^{d} \otimes_{A} A /(f) \\
\| & & \| \\
\operatorname{Sym}_{A /(f)}^{d}(I / f \cdot I) & & I^{d} / f \cdot I^{d} \\
\| & & g \downarrow \\
\operatorname{Sym}_{A /(f)}^{d}(I /(f) \cap I) & \stackrel{h}{\rightarrow} & I^{d} /(f) \cap I^{d}
\end{array}
$$

in which all of the maps are surjections. As a result $h$ is an isomorphism if and only if $l$ and $g$ are each isomorphisms. $g$ is an isomorphism if and only if the image of $f$ in $A / I^{d}$ is a nonzero divisor. Thus (2) implies (1) and to show that (1) implies (2) we need only demonstrate that if $l$ is an isomorphism then the map $\operatorname{Sym}_{A}^{d}(I) \rightarrow I^{d}$ is an isomorphism. Let $K$ be the kernel of this map. Since $f$ is not a zero divisor the sequence $0 \rightarrow K \rightarrow \operatorname{Sym}_{A}^{d}(I) \rightarrow I^{d} \rightarrow 0$ remains exact after tensoring with $A /(f)$. This shows that if $l$ is an isomorphism then $K$ and $f \cdot K$ are equal, which by Nakayama's lemma completes the proof. 
Theorem A.3. Let

$$
X \stackrel{i}{\hookrightarrow} Y \stackrel{j}{\hookrightarrow} Z
$$

be a composition of embeddings. If $i$ is linear and $j$ is regular then the composition $j \circ i$ is linear.

Proof. If $Z$ is a vector bundle and $j$ is the zero section then the result follows from Corollary A.2 (and induction on the rank of the bundle). In general we make use of the deformation to the normal cone to reduce to this case. As is the proof of Theorem 2 by [F, p. 64] there is a scheme $S$ with a flat map $S \stackrel{\pi}{\rightarrow} \mathbf{A}^{1}$ and an embedding $X \times \mathbf{A}^{1} \stackrel{K}{\leftrightarrow} S$ commuting with the maps to $\mathbf{A}^{1}$ with the following properties:

The open set $U=\pi^{-1}\left(\mathbf{A}^{1} \backslash\{0\}\right)$ is isomorphic to the product $Z \times\left(\mathbf{A}^{1} \backslash\{0\}\right)$ and the embedding $X \times\left(\mathbf{A}^{1} \backslash\{0\}\right) \hookrightarrow U$ is the composition

$$
X \times\left(\mathbf{A}^{1} \backslash\{0\}\right) \hookrightarrow Y \times\left(\mathbf{A}^{1} \backslash\{0\}\right) \hookrightarrow Z \times\left(\mathbf{A}^{1} \backslash\{0\}\right)
$$

The fibre over $0, S_{0}$ is isomorphic to the normal bundle of $Y$ in $Z$ and the embedding $K_{0}$ is the composition

$$
X \stackrel{i}{\hookrightarrow} Y \hookrightarrow N_{Y} Z
$$

(where the last embedding is the zero section). Fix an integer $d$ and a point $x \in X$. We will show that the surjection $\operatorname{Sym}_{\mathscr{O}_{Z, x}}^{d}\left(I_{x}\right) \rightarrow I_{x}^{d}$ is an isomorphism. Let $J \subset \mathscr{O}_{S}$ be the ideal sheaf of $X \times \mathbf{A}^{1}$. Let $f_{t}$ be a local parameter for $\mathbf{A}^{1}$ at the point $t$ and let $p$ be the point $K(x, 0) . f_{t}$ is a nonzero divisor in $\mathscr{O}_{S, p}$ and its image in $\mathscr{O}_{S, p} / J_{p}$ is again a nonzero divisor. By the remarks at the beginning of the proof the embedding $X \times\{0\} \hookrightarrow S_{0}$ is linear and thus in particular the surjection

$$
\operatorname{Sym}_{\mathscr{O}, p / f_{0}}^{d}\left(J_{p} / f_{0} \cap J_{p}\right) \rightarrow J_{p}^{d} / f_{0} \cap J_{p}^{d}
$$

is an isomorphism. By Lemma A.5 this implies that the surjection $\operatorname{Sym}_{\mathscr{O} S, p}^{d}\left(J_{p}\right)$ $\rightarrow J_{p}^{d}$ is an isomorphism. The same result then holds for all points in a neighborhood of $p$ and in particular for some $q=K(x, t)$ with $t$ nonzero we have

$$
\operatorname{Sym}_{\mathscr{O}_{S, q}}^{d}\left(J_{q}\right) \rightarrow J_{q}^{d}
$$

Now $f_{t}$ is not a zero divisor in $\mathscr{O}_{S, q}$ and its image is not a zero divisor in $\mathscr{O}_{S, q} / J_{q}^{d}$. (In $U S$ is a product so that the subscheme defined by $J^{d}$ is the product $\bar{X} \times \mathbf{A}^{1} \backslash\{0\}$ where $\bar{X}$ is the subscheme of $Z$ defined by $I^{d}$. In particular $J^{d}$ defines a subscheme of $U$ flat over $\mathbf{A}^{1}$.) Thus by Lemma A.5 the surjection

$$
\operatorname{Sym}_{\mathscr{O}_{s, q / f_{t}}^{d}}\left(J_{q} / f_{0} \cap J_{q}\right) \rightarrow J_{q}^{d} / f_{t} \cap J_{q}^{d}
$$

is an isomorphism. But (since $U$ is a product) this is the map

$$
\operatorname{Sym}_{\mathscr{O}_{Z, x}}^{d}\left(I_{x}\right) \rightarrow I_{x}^{d} \text {. }
$$
$F$.

We will say that a cone is linear if it is $\operatorname{Spec} \operatorname{Sym}(F)$ for some coherent sheaf

Lemma A.6. Any section of a linear cone is a linear embedding. 
Proof. There is an automorphism of the cone carrying the section to the zero section (subtract off the section), thus we reduce to the case of the zero section. One now checks the result immediately using Theorem A.2.

Theorem A.4. Let

$$
X \stackrel{i}{\rightarrow} Y \stackrel{j}{\rightarrow} Z
$$

be a composition of embeddings. If $i$ is regular and $j \circ i$ is linear then $j$ is linear along $X$.

Proof. As in the proof of Theorem A.3 we make use of the deformation to the normal cone. There exist schemes $S$ and $T$ with flat maps $S \stackrel{\pi}{\rightarrow} \mathbf{A}^{1}$ and $T \stackrel{p}{\rightarrow} \mathbf{A}^{1}$ and a composition of embeddings $X \times \mathbf{A}^{1} \hookrightarrow T \hookrightarrow S$ commuting with the projections to $\mathbf{A}^{1}$ and with the properties:

Over the open set $\mathbf{A}^{1} \backslash\{0\}$ this is the composition

$$
X \times \mathbf{A}^{1} \backslash\{0\} \hookrightarrow Y \times \mathbf{A}^{1} \backslash\{0\} \hookrightarrow Z \times \mathbf{A}^{1} \backslash\{0\}
$$

while on the fibres over zero we have the composition

$$
X \hookrightarrow N_{X} Y \hookrightarrow C_{X} Z \text {. }
$$

The embedding of $N_{X} Y$ in $C_{X} Z$ is induced by the surjection $I / I^{2} \rightarrow I / I^{2}+J$. Since the term on the right is locally free, this locally splits, and thus locally $C_{X} Y$ is a linear cone over $N_{X} Y$ and the above embedding is a section. Hence by the previous lemma, this embedding is linear, i.e. $p^{-1}(0) \hookrightarrow \pi^{-1}(0)$ is a linear embedding. This implies, just as in the proof of Theorem A.2, that for nonzero $t$ the embedding $p^{-1}(t) \hookrightarrow \pi^{-1}(t)$ is linear along $X \times\{t\}$. Since this is the embedding of $Y$ in $Z$, this completes the proof.

\section{REFERENCES}

[F] W. Fulton, Intersection theory, Springer-Verlag, New York, 1981.

[S] S. Herzog and K. Vasconcelos, Homology and blowing up rings, Commutative Algebra, Lecture Notes in Pure and Appl. Math., Springer-Verlag, 1983, pp. 79-169.

[H] C. Huneke, On the symmetric algebra and Rees algebra of an ideal generated by $a d$ sequence, J. Algebra 62 (1980), 268-275.

_, Determinantal ideals of linear type, Arch. Math. 47 (1986), 324-329.

__, Linkage and the Koszul homology of ideals, Amer. J. Math. 104 (1982), 1043-1062.

[K1] S. Keel, Intersection theory of polygon spaces (preprint).

[K2] - Intersection theory of the moduli space of pointed rational curves (preprint).

[KL] S. L. Kleinman, Multi-point formulas I: iteration, Acta Math. 147 (1978), 230-266.

[Ko] B. Kotsev, Determinantal ideals of linear type of a generic symmetric matrix (preprint).

[Mi] Micali, Sur les algebre universelles, Ann. Inst. Fourier (Grenoble) 14 (1964), 33-38.

[Mu] D. Mumford, Lectures on curves on an algebraic surface, Princeton Univ. Press, Princeton, N.J.,

[V] G. Valla, On the symmetric and Rees algebra of an ideal, Manuscripta Math. 30 (1980), 239-255.

Department of Mathematics, University of Utah, Salt lake City, Utah 84112

Current address: Department of Mathematics, University of Texas at Austin, Austin, Texas 78712

E-mail address: keel@utexas.edu 